\title{
Recognition of Classes and Types \\ of Air Objects on Two-Dimensional Radar its Images in the Surveyed Radar
}

\author{
Valeriy P. Berdyshev*a, Oleg N. Pomazuev', \\ Aleksei N. Saveliev ${ }^{c}$, Michael A. Smolkind, \\ Vladimir A. Kopylov ${ }^{\mathrm{e}}$ and Vitalii V. Loy \\ ${ }^{a}$ Military Academy of Aero-Space Defense \\ named after the Marshal of Soviet Union G.K. Zhukov \\ 50 Zhigareva Str., Tver, 170022, Russia \\ ${ }^{b}$ Main Department of Scientific and Research Activities \\ and Technological Support of the Advanced Technologies \\ of the Ministry of Defense of the Russian Federation \\ 84/32 Profsojznaj Str., Moscow, 117997, Russia \\ "JSC "Airborne navigation systems" \\ 15, 12 Bolshaya Novodmitrovskaya Str., Moscow, 127015, Russia \\ "Air Force and Air Defense Army" \\ 4 Dvortsovaya Square Str., St. Petersburg, 191186, Russia \\ eSiberian Federal University \\ 79 Svobodny, Krasnoyarsk, 660041, Russia
}

Received 07.09.2017, received in revised form 21.03.2018, accepted 11.11.2018

Currently, the task of recognizing air objects is of increasing interest, especially for air traffic control and air defense system developers, since the realization of recognition modes ensures an increase in the adequacy of radar information received from radars at control points of various degrees of hierarchy in an actual situation, decisions made, and also to automate the process of their adoption and create the conditions for the introduction of elements of an action sstvennogo intelligence in the operation of the relevant systems.

The article is devoted to an estimation of quality of recognition of classes and types of air objects in survey radars. Recognition is carried out by statistical methods using the features extracted from two-dimensional radar images, using the known method of generalized voting. Recognition features are polygonal area formed by a two-dimensional image; the number of resolved scattering centers on two-dimensional images; distance between the most distant scattering centers; the effective scattering surface, as the sum of the effective scattering surface of all scattering centers.

The results of the evaluation of the quality of class recognition (large, medium and small-sized goal) and types in classes of large and medium-sized objectives are presented.

(C) Siberian Federal University. All rights reserved

This work is licensed under a Creative Commons Attribution-NonCommercial 4.0 International License (CC BY-NC 4.0).

* Corresponding author E-mail address: berd696969@mail.ru, 1v2009.1974@mail.ru 
The obtained results can be used for developing and assessing the quality of class recognition systems and types of air assets based on signal attributes in existing and prospective radars, as well as by the person making the decision when choosing a radar recognition system and comparing alternative options.

Keywords: algorithm, face, portrait, portrait of dalnostnyj radar, azimuthal portrait, two-dimensional radar imageapplication, of inverse aperture synthesis, multi-frequency probe signal, detection rate.

Citation: Berdyshev V.P., Pomazuev O.N., Saveliev A.N., Smolkin M.A., Kopylov V.A., Loy V.V. Recognition of classes and types of air objects on two-dimensional radar its images in the surveyed radar, J. Sib. Fed. Univ. Eng. technol., 2019, 12(1), 18-29. DOI: 10.17516/1999-494X-0102.

\title{
Распознавание классов и типов воздушных объектов по двумерным радиолокационным изображениям в обзорной РЛС
}

\author{
В.П. Бердышев ${ }^{a}$, О.Н. Помазуев ${ }^{\sigma}$, А.Н. Савельев \\ М.А. Смолкин ${ }^{\text {, }, ~ В . А . ~ К о п ы л о в ~}$ \\ ${ }^{a}$ Военная академия воздушно-космической обороны \\ им. Маршала Советского Союза Г.К. Жукова \\ Россия, 170022, Тверь, ул. Жигарева, 50 \\ ${ }^{б}$ Главное управление научно-исследовательской деятельности \\ и технологического сопровождения передовых технологий МО РФ \\ Россия, 117997, Москва, ул. Профсоюзная, 84/32 \\ ${ }^{6} \mathrm{OAO}$ «Бортовые аэронавигаиионные системы» \\ Россия, 127015, Москва, ул. Большая Новодмитровская, 12, стр. 15 \\ ¿"Армия ВВС и ПВО» \\ Россия, 191186, Санкт-Петербург, ул. Двориовая площадь, 4 \\ ${ }^{\circ}$ Сибирский федеральный университет \\ Россия, 660041, Красноярск, пр. Свободный, 79
}

В настоящее время задача распознавания воздушных объектов вызывает повышенный интерес, особенно у разработчиков систем управления воздушным движением и противовоздушной обороны, поскольку реализация режимов распознавания обеспечивает повымение адекватности радиолокационной информации, получаемой от РЛС на пунктах управления различной степени иерархии, в реально складывающейся обстановке, что позволяет оптимизировать принимаемые решения, а также автоматизировать процесс их принятия и создавать условия для введения элементов искусственного интеллекта в управление соответствуюших систем. Статья посвящена оченке качества распознавания классов и типов воздушных объектов в обзорных РЛС. Распознавание проводится статистическими методами с использованием признаков, извлеченных из двумерных радиолокачионных изображений, с использованием известнойметодики обобщенного голосования. Признаками распознавания являются: площадь многоугольной фигуры, образованной двумерным изображением; количество разрешаемых рассеивающих иентров на двумерных изображениях; расстояние между наиболее удаленными рассеивающими иентрами; эффективная поверхность рассеяния как сумма эффективной поверхности рассеяния всех рассеивающих цеентров. 
Представлень результаты оценки качества распознавания классов (крупно-, средне- и малоразмерная цель) и типов в классах крупно- и среднеразмерная цель.

Полученные результаты могут быть использованы специалистами при разработке и оценке качества систем распознавания классов и типов воздушных объектов по сигнальным признакам в существующих и перспективных РЛС, а также лицуом, принимающим решение при выборе системы распознавания РЛС и сравнении альтернативных вариантов.

Ключевые слова: алгоритм, распознавание, радиолокационный портрет, дальностный портрет, азимутальный портрет, двумерное радиолокационное изображение, инверсное синтезирование апертуры, многочастотный зондирующий сигнал, вероятность распознавания.

Одним из основных направлений развития систем и средств управления воздушным движением (УВД) и противовоздушной обороны (ПВО) является расширение их возможностей и повышение качества радиолокационной информации. Информация распознавания применительно к РЛС обзора может быть использована для решения следующих задач: вскрытия состава групповых целей; выбора наиболее важных целей из состава налета; селекции малоподвижных ложных целей (ЛЦ), например атмосферных образований типа «ангел-эхо», стай птиц и т.п.; селекции тактических и баллистических ЛЦ; адаптации системы вторичной обработки к маневренным возможностям целей; селекции многократных ответных имитирующих помех и др. $[1,2]$.

Реализация режимов распознавания воздушных объектов (ВО) обеспечивает повышение адекватности радиолокационной информации (РЛИ), получаемой от РЛС на пунктах управления различной степени иерархии, в реально складывающейся обстановке, что позволяет оптимизировать принимаемые решения, а также автоматизировать процесс их принятия и создавать условия для введения элементов искусственного интеллекта в управление соответствующих систем УВД и ПВО. Кроме того, использование информации распознавания дает возможность существенно повысить эффективность контроля воздушного пространства. Информация распознавания должна быть получена на максимальных дальностях обнаружения ВО в реальном масштабе времени с максимально возможной достоверностью.

Разработка систем распознавания, в частности радиолокационного распознавания, сопряжена с решением целого ряда задач. Центральным является вопрос о словаре признаков, на языке которых производятся как априорные описания классов ВО, так и апостериорное описание распознаваемых объектов. Связано это с тем, что рабочий словарь признаков определяет состав и технические характеристики измерительных средств системы распознавания, в данном случае РЛС, которые признаны наиболее ресурсоемкими элементами системы.

Распознавание ВО может быть реализовано: по тактическим признакам; по сигнальным признакам; по радиоизлучению ВО; по траекторным признакам; по комплексным характеристикам ВO [1-3].

В качестве тактических признаков обычно используются: тактическая дальность; курс цели относительно линии боевого соприкосновения (ЛБС); курсовой параметр цели относительно разведанных аэродромов противника и стартовых площадок ракет; курсовой параметр цели относительно стыка зон ответственности (обнаружения) различных средств ПВО. Тактические признаки характеризуют решаемые СВН задачи, возможный количественный состав

$$
-20-
$$


групп целей, способы постановки помех, боевые порядки СВН, способы применения бортового оружия с воздушных носителей, наиболее вероятные маршруты полета и т.д.

К сигнальным признакам относятся: интенсивность принимаемых сигналов на одной или разных частотах, т.е. величина эффективной поверхности рассеяния (ЭПР) ВО; флюктуация ЭПР ЛА, а также спектр флюктуаций ЭПР; импульсная характеристика ВО, а также его передаточная характеристика, полученная путем Фурье-преобразования импульсной характеристики либо излучения серии дискретных сигналов со спектром частот от 0 до $\infty$ (на практике число дискретных частот выбирают порядка 10); собственные резонансы ЛА (измеряют фазовые сдвиги, вносимые ВО при его облучении двумя когерентными гармоническими сигналами кратных частот); поляризационные характеристики отраженных одночастотных и многочастотных сигналов; структура сжатого широкополосного сигнала, представляющая собой одномерное радиолокационное изображение ВО (радиолокационный дальностный портрет); двумерные радиолокационные изображения (ДРЛИ); характеристики «турбинной» модуляции и др.

К траекторным признакам относятся: скорость, курс и высота полета воздушных объектов, развиваемые максимальные ускорения; маневренные характеристики сопровождаемых объектов; энергетическая высота $\left(H_{\ni}=V^{2} / 2 g+H\right.$, где $V$ - скорость движения $\mathrm{BO}, g$ - ускорение свободного падения, $H$ - высота полета ВО) и скорость изменения энергетической высоты $\left(V_{H}\right)$; особенности поведения цели и другие признаки.

В [4] предложена имитационная математическая модель, а в [5] - программа, реализующая ее работу при построении и исследовании радиолокационных портретов ВО в интересах распознавания статистическими методами с использованием признаков, извлеченных из ДРЛИ. В основу модели положены результаты, представленные в [6-10].

На рис. 1 отражены результаты моделирования дальностных портретов (ДП) (а, г, ж), азимутальных портретов (АП) $(б$, д, з) и двумерных радиолокационных изображений (ДРЛИ) (в, е, и) при ракурсе локации q=150 целей типа В-52, А-10А и ДПЛА Predator соответственно [5].

При моделировании вводили следующие исходные данные: ракурсы целей $-15^{\circ}$, дальность - 30000 м; высота полета - 1000 м; скорость полета - 200 м/с; амплитуда ТН - 2॰ , угловая скорость поворота за счет ТН - 1,5\%; вид движения цели - равномерное вращение вокруг ЦСЦ с угловой скоростью; несущая частота РЛС - 10000 МГц; количество частот МЧЗС - 128; диапазон перестройки частоты 150 МГц; амплитуда внутренних шумов приемника - 0 усл. ед.

Анализ полученных при моделировании радиолокационных портретов (РЛП) ВО различных классов показывает, что они позволяют с высокой достоверностью оценивать особенности пространственной структуры и геометрические размеры целей, летящих в условиях траекторных нестабильностей (ТН). Это позволило предположить, что признаки, заключенные в дальностных, азимутальных портретах и в ДРЛИ ВО, могут быть эффективно применены в перспективных устройствах распознавания. Проверка этой гипотезы и оценка качества распознавания будут проведены статистическими методами с использованием признаков, извлеченных из ДРЛИ.

Цель статьи - применить известную методику обобщенного голосования и оценить результаты распознавания классов и типов ВО по двумерным радиолокационным изображениям (ДРЛИ) в обзорной РЛС с многочастотными зондирующими сигналами (МЧЗС) и инверсным радиолокационным синтезированием апертуры (ИРСА). 


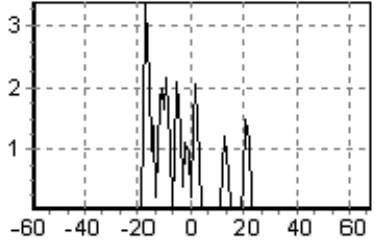

a)

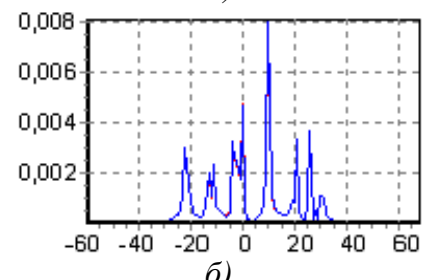

б)

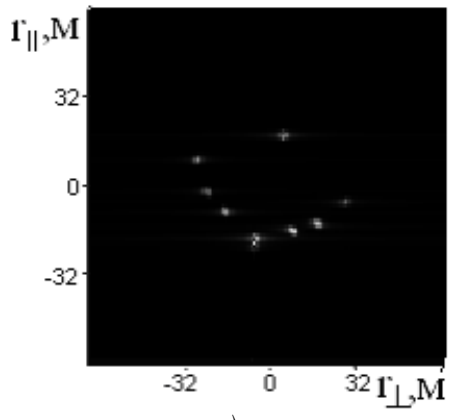

в)

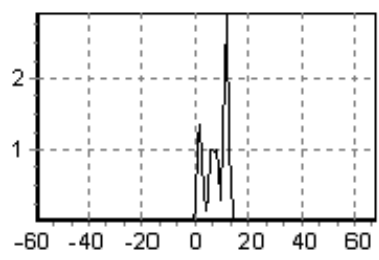

2)

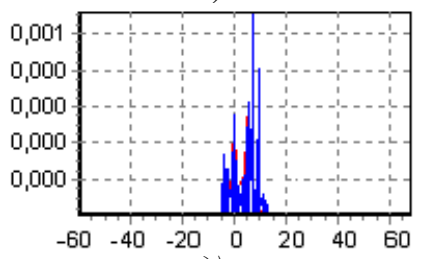

d)

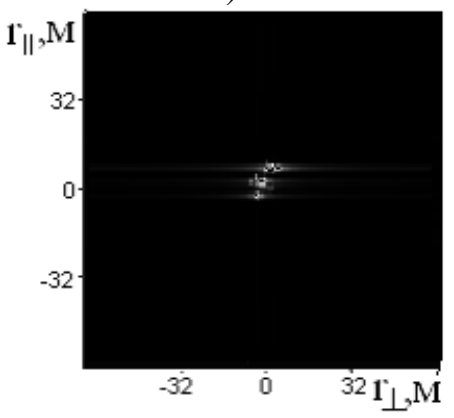

e)
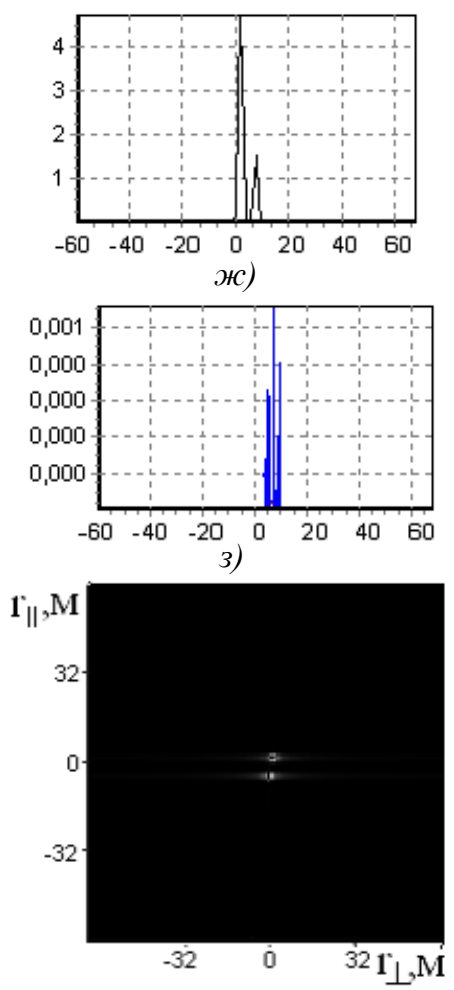

u)

Рис. 1. Радиолокационные портреты воздушных целей

Fig. 1. Radar portraits of air targets

В соответствии с целевым назначением к имитационной математической модели (ИММ) предъявлялись следующие требования: возможность проведения исследований при любых ракурсах локации; возможность имитации движения цели по различным траекториям (высота, скорость, дальность) при наличии помех и внутренних шумов приемника различного уровня. На рис. 2 показан вид интерфейса ввода исходных данных и радиолокационных портретов воздушных объектов.

Применим известную методику обобщенного голосования для оценки результатов распознавания классов и типов ВО по двумерным радиолокационным изображениям в обзорной РЛС с МЧЗС и ИРСА. Основные этапы методики распознавания следующие [3].

1. Выявление признаковой совокупности. Изучение особенностей различных признаков позволило для проведения дальнейших исследований и получения оценок распознавания предложить следующую признаковую совокупность (рис. 32): площадь многоугольной фигуры, образованной ДРЛИ, - S; количество разрешаемых РЦ на ДРЛИ - М; расстояние между наиболее удаленными РЦ - R; ЭПР как сумма ЭПР всех РЦ - $\sigma$.

2. Выбор метода распознавания. Для исследования информативности предложенных выше признаков ДРЛИ проведено распознавание аэродинамических ВЦ методом математического моделирования. 


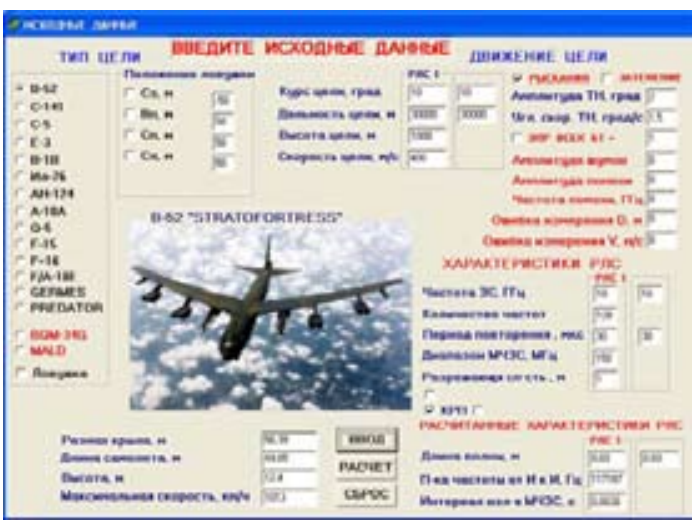

a)

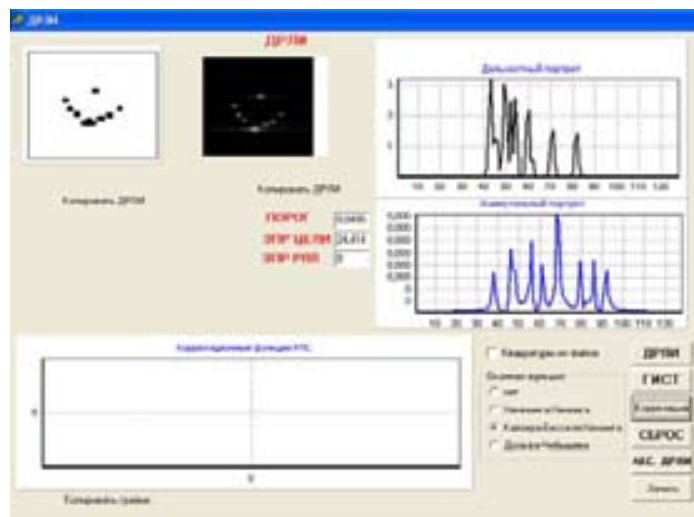

б)

Рис. 2. Вид интерфейса ввода исходных данных (а) и радиолокационных портретов воздушных объектов (б)

Fig. 2. Type of input interface for input data (a) and radar portraits of air units (б)

Таблица 1. Классы целей, различающиеся геометрическими размерами

Table 1. Classes of goals that differ in geometric dimensions

\begin{tabular}{|c|c|c|c|c|c|c|c|c|}
\hline \multirow{3}{*}{ Класс цели } & \multirow{3}{*}{ Тип цели } & \multirow{3}{*}{$\begin{array}{c}\text { Класс } \\
\text { тактический }\end{array}$} & \multicolumn{2}{|c|}{ Размеры цели } & \multicolumn{4}{|c|}{ Средние размеры класса } \\
\hline & & & \multirow[t]{2}{*}{ Длина, м } & \multirow{2}{*}{$\begin{array}{c}\text { Размах } \\
\text { крыльев, м }\end{array}$} & \multicolumn{2}{|c|}{ Длина, м } & \multicolumn{2}{|c|}{$\begin{array}{c}\text { Размах } \\
\text { крыльев, м }\end{array}$} \\
\hline & & & & & мин & макс & мин & макс \\
\hline \multirow{5}{*}{ КРЦ } & $\mathrm{C}-5$ & \multirow{2}{*}{ BTA } & 75,54 & 67,88 & \multirow{5}{*}{45} & \multirow{5}{*}{76} & \multirow{5}{*}{24} & \multirow{5}{*}{68} \\
\hline & C-141 & & 51,29 & 48,74 & & & & \\
\hline & B-52 & \multirow{2}{*}{$\mathrm{CA}$} & 49,05 & 56,39 & & & & \\
\hline & B-1B & & 44,81 & $41,67 / 23,84$ & & & & \\
\hline & E-3C & ДРЛО & 46,61 & 44,42 & & & & \\
\hline \multirow{5}{*}{ СРЦ } & F-18E & \multirow{4}{*}{ TA } & 18,31 & 11,43 & \multirow{5}{*}{15} & \multirow{5}{*}{19} & \multirow{5}{*}{9} & \multirow{5}{*}{18} \\
\hline & F-15 & & 19,05 & 13,05 & & & & \\
\hline & F-16 & & 15,03 & 9,45 & & & & \\
\hline & Q-5 & & 15,65 & 9,68 & & & & \\
\hline & A-10A & AA & 16,26 & 17,53 & & & & \\
\hline \multirow{3}{*}{ МРЦ } & Germes & \multirow{3}{*}{ ДПЛА } & 15 & 9 & \multirow{3}{*}{5} & \multirow{3}{*}{15} & \multirow{3}{*}{1} & \multirow{3}{*}{9} \\
\hline & Predator & & 14.84 & 8,23 & & & & \\
\hline & BQM & & 4,58 & 1 & & & & \\
\hline
\end{tabular}

3. Определение алфавита классов. Алфавит классов целей состоял из 3-х классов: крупноразмерная (КРЦ), среднеразмерная (СРЦ) и малоразмерная цель (МРЦ). С учетом геометрических размеров ЛА (табл. 1) вся совокупность целей из 13 типов ЛА была распределена по трем указанным классам.

4. Задание диапазонов изменения параметров целей и РЛС при распознавании. При моделировании ДРЛИ различных ВЦ строились на ракурсах из диапазона курсовых углов $\pm 30^{\circ}$ и углов места $2^{0} \ldots 20^{0}$ с дискретностью $0,1^{0}$. Моделировалось движение цели в условиях траектор- 
ных нестабильностей на высотах $1000 \ldots 10000$ м, на дальностях $10 \ldots 50$ км, со скоростями полета $200 \ldots 700$ м/с, с максимальной угловой скоростью рысканий $0,5 \ldots 2,5 \%$. Диапазон перестройки частоты составлял 150 МГц. Количество частот МЧЗС принималось равным 128. Период повторения $\mathrm{T}_{\text {и }}=30$ мкс. Отношение сигнал/шум изменялось в диапазоне $-2 \ldots 4$ дБ. Учитывалась возможность затенения РЦ элементами конструкции планера. Ошибки измерения дальности достигали величины 50 м. Ошибки измерения скорости не превышали 2 м/с.

5. Выявление законов распределения признаков, по которым будет решаться задача распознавания и построение их гистограмм. Для этого при формировании обучающей выборки случайным образом выбираются конкретные значения параметров в пределах указанных выше диапазонов. Аналогично случайным образом выбирается один из пяти типов целей, входящих в класс КРЦ и СРЦ, и один из 3-х типов, входящих в класс МРЦ. Для классов КРЦ и СРЦ было проведено по 500 опытов, а для МРЦ - соответственно 300 опытов. На рис. 3 представлены гистограммы распределения 4-х предложенных признаков: «количество разрешаемых РЦ» (a), «площадь многоугольной фигуры, образованной ДРЛИ» (для классов КРЦ и СРЦ) (б), «максимальное расстояние между РЦ» (в) и «ЭПР» (г) для классов КРЦ, СРЦ, МРЦ.

На рис. 3 видно, что все признаки распознавания являются информативными. Поскольку число РЦ в классе МРЦ не превышало двух, то признак $\mathrm{S}$ для этого класса не существует. Поэтому на рис. $3 б$ класс МРЦ не представлен. При распознавании для МРЦ значение признака $\mathrm{S}$ всегда принималось равным нулю. На рис. 4 изображены гистограммы распределения признаков «площадь многоугольной фигуры, образованной ДРЛИ» (а), «количество разрешаемых РЦ» (б), «максимальное расстояние между РЦ» (в) и «ЭПР» (г) для типов целей С-5, C-141, В-52, Е-3 и В-1В, а на рис. 5 - признаки «площадь многоугольной фигуры, образованной ДРЛИ» (a), «количество разрешаемых РЦ» (б), «максимальное расстояние между РЦ» (в) и «ЭПР» (г) для

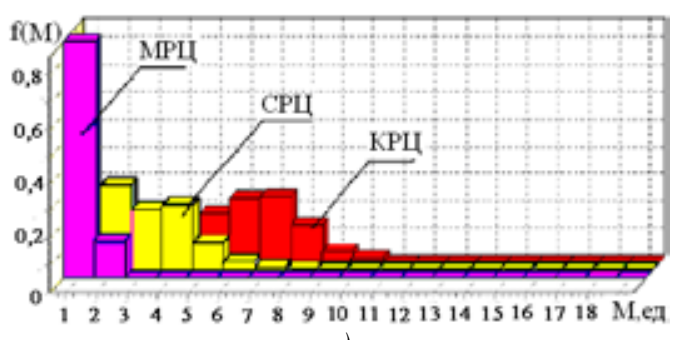

a)

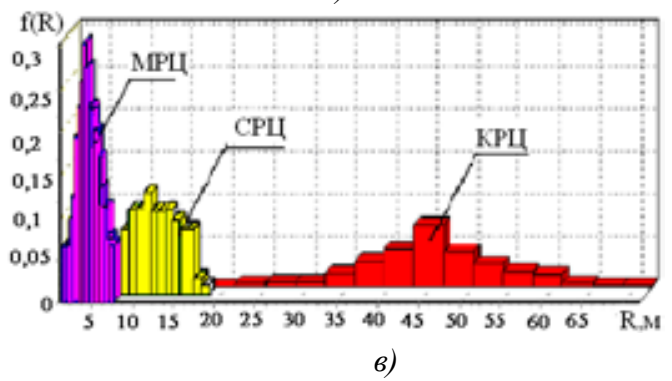

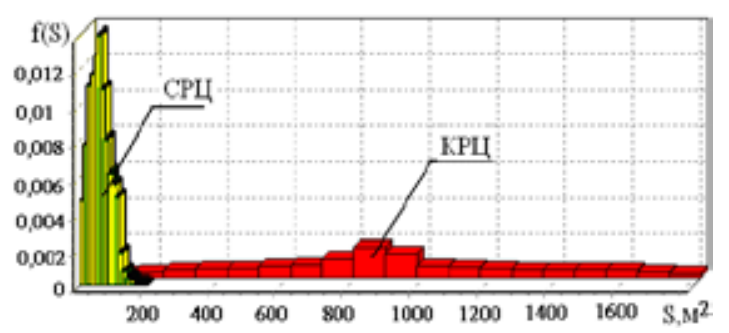

б)

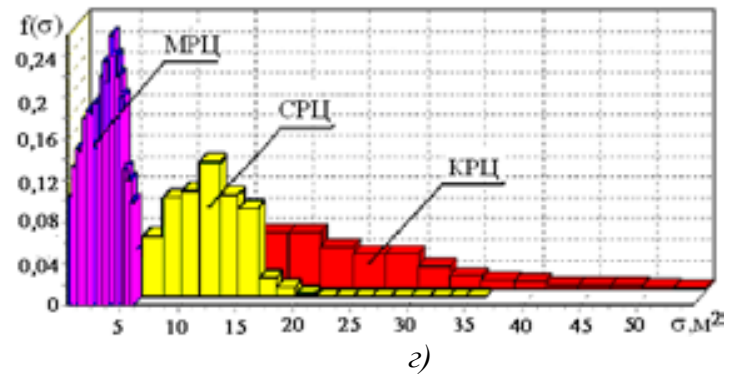

Рис. 3. Гистограммы распределения признаков для классов целей

Fig. 3. Histogram distribution of signs for purposes of classes 


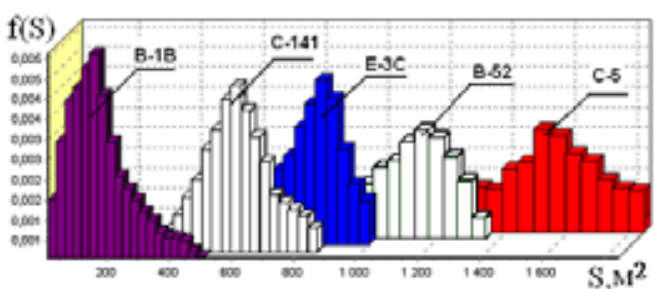

a)

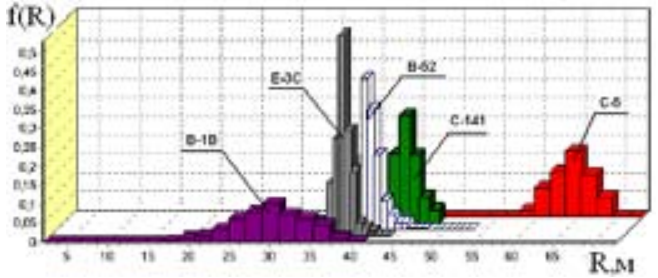

8)

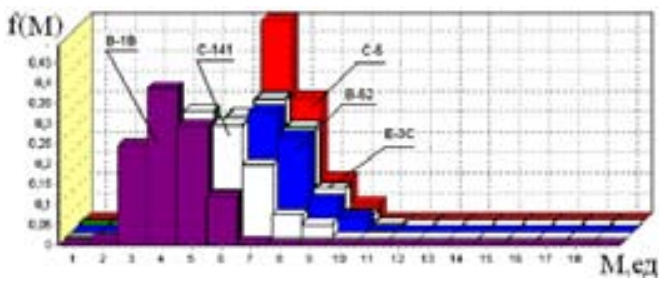

б)

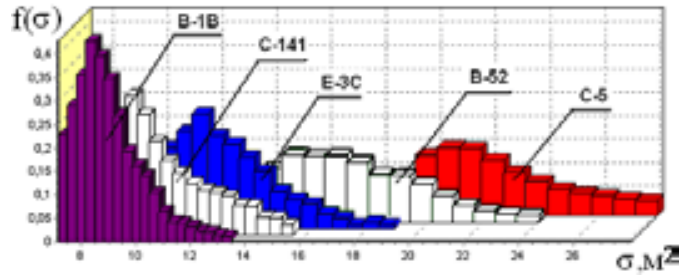

2)

Рис. 4. Гистограммы распределения признаков для типов целей С-5, C-141, B-52, Е-3 и В-1B

Fig. 4. Histogram distribution of signs for the types of C-5, C-141, B-52, E-3 and B-1B

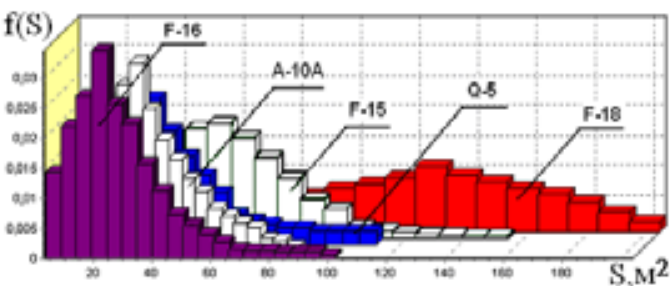

a)

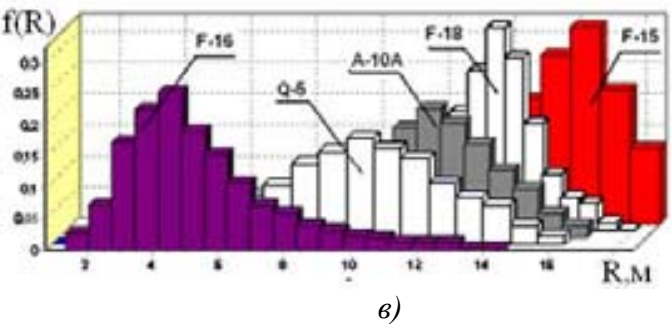

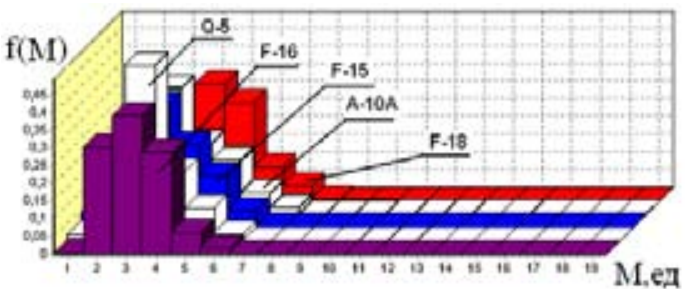

б)

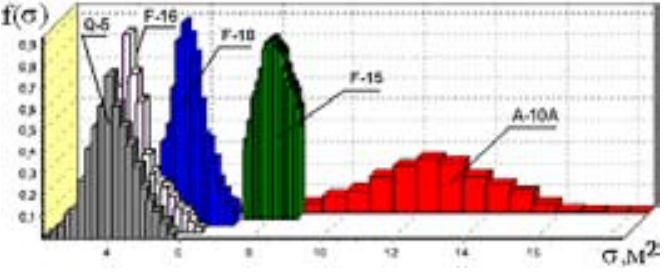

2)

Рис. 5. Гистограммы распределения признаков для типов целей F-18, F-15, Q-5, A-10A и F-16

Fig. 5. Histogram distribution of signs for the types of goals the F-18, F-15, Q-5, A-10A and F-16

типов целей F-18, F-15, Q-5, A-10A и F-16 для распознавания типов целей в классе КРЦ и классе СРЦ соответственно.

Из полученных гистограмм видно, что для распознавания типов целей в классе КРЦ признак «количество разрешаемых РЦ» не является информативным. Поэтому распознавание типов в классе КРЦ предложено проводить с использованием не 4-х, а 3-х признаков. В классе СРЦ наибольшей информативностью обладают признаки «максимальное расстояние между РЦ» и «ЭПР». Поэтому в алгоритмах распознавания типов в классе СРЦ целесообразно использовать только два этих признака. 
6. Сглаживание полученных гистограмм по методу наименьиих квадратов полиномами $7 . . .16$ степеней. Решение задачи распознавания при моделировании алгоритмически удобнее проводить на основе аналитических выражений для плотности распределения признака. При этом полученная в опыте реализация признака не нуждается в многочисленных сравнениях с порогами разрядов (кластеров) для нахождения оценки вероятности. Величина признака используется в качестве аргумента функции, выражающей плотность вероятности признака. Это придает алгоритмам распознавания стройность, простоту, удобство проведения анализа и т.д. При этом в роли оценок вероятностей отнесения цели к определенному классу по какому-либо признаку целесообразно использовать оценку вероятности попадания признака на интервал, величина которого выбирается равной половине ширины наименьшего разряда гистограмм, используемых для получения плотностей распределения по данному признаку. Для реализации этого при подготовке ИММ и решению задачи распознавания было проведено сглаживание полученных гистограмм по методу наименьших квадратов полиномами 7...16 степеней.

В качестве примера приводим полином 10-й степени, аналитически выражающий плотность вероятности признака «максимальное расстояние между РЦ» для класса СРЦ:

$$
\begin{aligned}
& f(R)=-1,076302216974403 \cdot 10^{-9} \cdot R^{10}+1,059123987616364 \cdot 10^{-7} \cdot R^{9}- \\
& -4,468482752647992 \cdot 10^{-6} \cdot R^{8}++1,057025665535967 \cdot 10^{-4} \cdot R^{7}- \\
& -1,539678546357074 \cdot 10^{-3} \cdot R^{6}+1,429048777744766 \cdot 10^{-2} \cdot R^{5}- \\
& -8,465365839584320 \cdot 10^{-2} \cdot R^{4}+3,121973159482199 \cdot 10^{-1} \cdot R^{3}- \\
& -6,768032566000665 \cdot 10^{-1} \cdot R^{2}++7,797658528210624 \cdot 10^{-1} \cdot R- \\
& -3,639166557350961 \cdot 10^{-1} .
\end{aligned}
$$

Нахождение коэффициентов полиномов производилось с помощью встроенных процедур пакета MATLAB 6,5 [11]. Полученные плотности вероятностей достаточно точно передают особенности распределений признаков. Следовательно, оценки вероятностей распознавания с их использованием будут соизмеримы с оценками, полученными на основе ранее представленных гистограмм. Аналогично были получены плотности вероятности распределения признаков для распознавания типов целей в классе КРЦ и в классе СРЦ соответственно. Следует отметить, что плотности вероятностей позволяют косвенно оценить возможности распознавания определенных типов целей по предложенным признакам. Например, на рис. 4 видно, что по признаку $\mathrm{R}$ самолеты E-3C и B-52 распознать практически невозможно. Зато цели типа C-5 и B-1B по этому же признаку распознаются почти достоверно. Распознавание типов Е-3С и В-52 дает желаемый результат за счет привлечения в алгоритмы признаков $\mathrm{S}$ и $\sigma$, по которым плотности вероятностей расходятся на половину своих площадей, отражающих значение вероятности.

Еще сложнее обстоит дело с распознаванием типов в классе СРЦ (рис. 5). Графики для признаков $\sigma$ и $\mathrm{R}$ показывают достаточно сильное совпадение по ним многих типов целей. Тем не менее совместное использование двух признаков ( $\sigma$ и R) позволяет различать СРЦ. Так, если F-16 и Q-5 по признаку б почти совпадают, то по признаку R они имеют отличия. При этом ЛА Q-5 и A-10A по признаку $\sigma$ распознаются почти достоверно, хотя по признаку R они пересекаются. По признаку $\sigma$ должны распознаваться с достаточной вероятностью такие пары целей, как F-16 и A-10A, Q-5 и F-15, F-16 и F-15, Q-5 и A-10A.

7. Определение контрольной выборки. Для получения оценок вероятности распознавания классов целей с использованием предложенных признаков и правила обобщенного голосова-

$$
-26-
$$



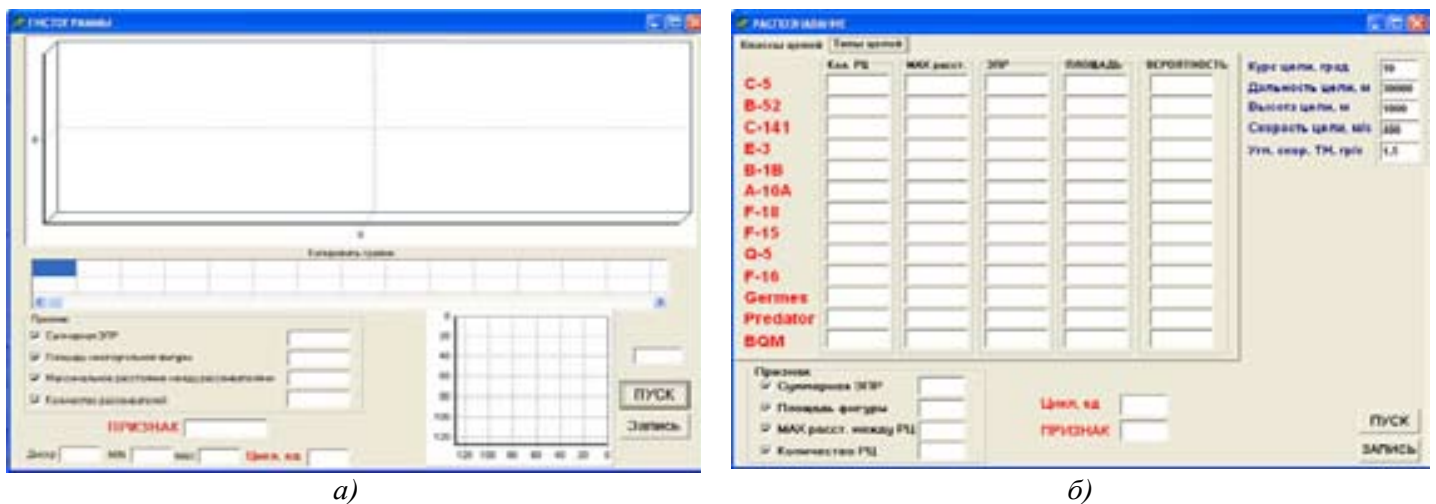

Рис. 6. Вид интерфейса построения гистограмм (а) и распознавания классов и типов воздушных объектов (б)

Fig. 6. View of the interface for constructing histograms (a) and recognizing the classes and types of air objects (б)

ния [3] было проведено распознавание исследуемых классов целей (КРЦ, СРЦ и МРЦ). Моделировалось получение ДРЛИ 13-ти исследуемых типов целей (см. табл. 1). Изображения всех ВЦ были получены при изменении по случайному закону параметров движения целей и характеристик РЛС в принятых диапазонах. Контрольная выборка насчитывала при распознавании по ДРЛИ 1300 опытов (по 500 - для КРЦ и СРЦ и 300 - для МРЦ).

Для получения количественных оценок распознавания методом моделирования по правилу обобщенного голосования в условиях априорной неопределенности решалась задача распознавания типов целей в рамках классов КРЦ и СРЦ. Контрольная выборка отличалась от предыдущей и насчитывала 1000 опытов (в среднем по 100 опытов на каждый тип цели).

8. Проведение моделирования с использованием предложенных признаков и правила обобщенного голосования. При моделировании были использованы перечисленные выше диапазоны изменения параметров целей и характеристик РЛС. На рис. 6 показан вид интерфейса построения гистограмм и распознавания классов и типов воздушных объектов.

9. Оиенка результатов моделирования. Оценка качества распознавания показала, что средняя вероятность правильного распознавания для классов КРЦ, СРЦ и МРЦ составляет: 0,95 (при отношении сигнал/шум 13 дБ) при средней вероятности ошибочного решения 0,05, доверительной вероятности 0,9 с шириной доверительного интервала 0,02 и 0,86 (при изменении отношения сигнал/шум в указанных пределах) при средней вероятности ошибочного решения 0,14 , доверительной вероятности 0,9 с шириной доверительного интервала 0,02 .

Средняя вероятность правильного распознавания типов целей составила: 0,87 (в классе КРЦ) при средней вероятности ошибочного решения 0,13 , доверительной вероятности 0,9 с шириной доверительного интервала 0,$08 ; 0,59$ (в классе СРЦ) при средней вероятности ошибочного решения 0,41 , доверительной вероятности 0,9 с шириной доверительного интервала 0,1 .

\section{Заключение}

Таким образом, с использованием методики обобщенного голосования получены оценки вероятности распознавания классов и типов воздушных объектов по двумерным радиолока-

$$
-27-
$$


ционным изображениям в обзорной РЛС с многочастотными зондирующими сигналами и инверсном радиолокационном синтезировании апертуры.

Полученные результаты могут быть использованы специалистами при разработке и оценке качества систем распознавания классов и типов ВО по сигнальным признакам в существующих (модернизируемых) и перспективных РЛС, а также лицом, принимающим решение, при выборе системы распознавания РЛС и сравнении альтернативных вариантов.

\section{Список литературы}

[1] В.П. Бердышев, Е.Н. Гарин, А.Н. Фомин и др. Радиолокационные системы / Под общей ред. В.П. Бердышева. Красноярск, Сиб. федер. ун-т, 2011. 400 с. [Radiolokacionnie sistemi / V.P. Berdyshev, V.P. Berdyshev, E.N. Garin, A.N. Fomin i dr./ Pod red. obcei red V.P. Berdysheva. Krasnoajrsk, Sib. feder.un-t, 2011, 400 p. (in Russia)]

[2] В.Н. Тяпкин, В.П. Бердышев, Е.Н. Гарин и др. Основы построения РЛС РТВ / Под общей ред. В.Н. Тяпкина. Красноярск, Сиб. федер. ун-т, 2011. 536 с. [V.N. Tajpkin, V.P. Berdyshev, E.N. Garin i dr. Osnovi postroeniaj RLS RTV / Pod red. obcei red V.N. Tajpkina. Krasnoajrsk, Sib. feder.un-t, 2011, 536 p. (in Russia)]

[3] Ширман Я.Д., Горшков С.А., Лещенко С.П, Орленко В.М. Методы радиолокационного распознавания и их моделирование. Зарубежная радиоэлектроника: Успехи современной радиоэлектроники. Киев. 1996, № 11, 3-62 [Shirman J.D., Gorshkov S.A., Leshchenko C.P., Orlenko V.M. Radar recognition Methods and modeling. Foreign Electronics: Achievements of modern electronics. Kiev. 1996. no. 11. pp. 3-62 (in Russia)]

[4] Миронов А.М., Митрофанов Д.Г., Помазуев О.Н., Бортовик В.В., Бердышев В.П., Цыганов А.А. Имитационная математическая модель построения и исследования радиолокационных портретов воздушных объектов в интересах распознавания. Свид. о регистр. электр. pecypca № 20498 от 19.11.2014 [Mironov A.M., Mitrophanov, D.G., Pomazuev O.N., Bortovik V.V., Berdishev V.P., Tsyganov A.A. Mathematical model building and Simulation study of radar portraits air objects for recognition. Certificate of registration of an electronic resource no. 20498, date of register. 19.11.2014 (in Russia)]

[5] Бердышев В.П., Миронов А.М., Помазуев О.Н., Стучилин А.И., Косолапов А.В. Программа, реализующая работу математической модели построения радиолокационных портретов воздушных объектов. Свид. о гос. регистр. прогр. для ЭВМ №2015615457 от 18.05.2015 [Berdishev V.P., Mironov A.M, Pomazuev O.N., Stuchilin A.I., Kosolapov, A.V. Program that implements the work of mathematical model building radar portraits air targets. State registration Certificate of a computer programs no. 2015615457 date of registering. 18.05.2015 (in Russia)]

[6] Стайнберг Б.Д. Формирование радиолокационного изображения самолета в диапазоне СВЧ. ТИИЭР. 1988. 76. 12. 26-46 [Steinberg B.D. Formation of the radar plane in the microwave frequency range. TIIJeR. 1988. 76. 12. 26-46. (in Russia)]

[7] Патент 2180445 (РФ) от 06.06.2000 г, МПК6 G 01 S 13/89. Способ построения двумерного радиолокационного изображения воздушной цели по траекторным нестабильностям ее полета / Митрофанов Д. Г., Гусев М.Д., Денисов А.В., Бортовик В. В. Заявка 2000114138/09. Приоритет 06.06.2000 г. [Patent 2180445 (RF), ot 06.06.2000 g, МПК6 G 01 S 13/89. Sposob postroeniaj dvumernogo radioloracionnogo izobrajeniaj vozduchnoi zeli po traektornim nestabilnostajm ee 
poleta / Mitrofanov D.G., Gusev M.D, Denisov A.V., Bortovik V.V. Zaajvka 2000114138/09. Prioritet 06.06.2000 (in Russia)]

[8] Патент 2234110 (РФ), МПК7 G 01 S 13/89. Способ построения двумерного радиолокационного изображения воздушной цели / Бортовик В.В., Митрофанов Д.Г. и др. [Patent 2234110 (RF), МПК7 G $01 \mathrm{~S}$ 13/89. Sposob postroeniaj dvumernogo radioloracionnogo izobrajeniaj vozduchnoi zeli / Bortovik V.V., Mitrofanov D.G. et al.]

[9] Митрофанов Д.Г., Силаев Н.В. Использование многочастотного узкополосного зондирующего сигнала для построения двумерного радиолокационного изображения объекта. $P a$ диоэлектроника. 2000. 40(12). 39-46 [Mitrofanov D.G., Silaev N.V. Ispolzovanie mnogocastotnogo uzkopolosnogo zondirucego signala dlaj postroeniaj dvumernogo radioloracionnogo izobrajeniaj obekta. Radioelektronika. 2000. 40(12). 39-46 (in Russia)]

[10] Бердышев В.П., Талалаев А.Б., Платонов А.Ю., Миронов А.М. Алгоритм вычисления и исследования функции неопределенности широкополосных когерентных многочастотных зондирующих сигналов, используемых для получения дальностных портретов в обзорных РЛС Программные продукты и системы. 2014. 3. 105-112 [Бердышев В.П., Талалаев А.Б., Платонов А.Ю., Миронов А.М. Алгоритм вычисления и исследования функции неопределенности широкополосных когерентных многочастотных зондирующих сигналов, используемых для получения дальностных портретов в обзорных РЛС. Программные продукты и системы. 2014. 3. 105-112 (in Russia)]

[11] Дьяконов В.П., Абрамова И.В. MATLAB. Обработка сигналов и изображений. Специальный справочник. СПб.: Питер, 2002. 608 с. [Deaconov V. P., Abramov I.V. MATLAB. Signal and image processing. A special handbook. Spb.: Piter, 2002. 608 p. (in Russia)] 